\title{
Scanning Electron Microscopy: Bridging the Gap from Stem Cells to Hydrogels.
}

\author{
L-M. Joubert*
}

* Cell Sciences Imaging Facility, Beckman Center, Stanford University Medical School, Stanford, CA 94305.

Stem cell research is no longer in its infancy. It has matured into a discipline with far-reaching applications. When little is known about a subject, the questions people raise are almost invariably ethical. As knowledge grows, however, people become amoral and more concerned with information i.e. more narrowly intellectual [1]. The words of Charles Spurgeon (1834-1992) that 'every generation needs regeneration' takes on new meaning in the era of stem cell biology [2] and there is a sense of urgency to move new developments quickly into the clinical setting. Visualization of stem cells is one of the core set of techniques that are applied to unlock the potential of stem cell biology. Various differentiation protocols revolve around fluorescence microscopy, applying different molecular markers. However, the limited resolution of light microscopy, and an inability to resolve surface features, has put Scanning EM in great demand to fill this gap. It is often also the preferred technique to apply before TEM investigation is started. The additional benefit of Variable Pressure (VP) SEM, enabling visualization of hydrated samples, e.g. biomaterials onto which stem cells are grafted before clinical application, has proved to be an indispensible tool in our recent research.

The ethical debate that ensued from Human Embryonic Stem cells (HESC) prompted large efforts to find alternative approaches to use adult stem cells or progenitor stem cells instead [3]. The concept that certain stem cells can trans-differentiate into cell types outside their normal lineage has been raised recently. We currently study adipogenic stem cells from humans, dogs and mice, and transform these into bone and other stem cells (Fig.1a-d). HESC cells generate cardiomyocytes, cells that contract rhythmically and will be engineered to restore contractile function of the heart. Light microscopic detection of 'beating' cells on glass coverslips are followed by SEM visualization, after marking the location of the (contracting) cells of interest. Otic progenitor cells (Fig.2a,b), i.e. cochlear stem cells, form floating colonies, so-called neurospheres that develop through different stages, from compact solid spheres into irregular partially hollow spheres and ultimately large hollow spheres, when cultured in non-adherent conditions [4]. Surface properties, cell junctions and inner and outer surface features are resolved by SEM, applying both secondary (SE) and backscatter electron (BSE) detection.

For transplantation or grafting of stem cells, biomaterials, hydrogels and various protein or polysaccharide scaffolds are developed. The structure and porosity of these scaffolds in their natural hydrated state are visualized by VP-SEM, with coolstage control of hydration [5]. Control of porosity is imperative for diffusion of growth media and proliferation of cells. Stem cells grown on such scaffolds are aldehyde-fixed and post-fixed in OsO4 to increase contrast, thereby enabling detection of fully hydrated cells on the hydrogel surface (Fig.2c). Low voltage SEM is in great demand for visualization of surface features not resolved by light or fluorescence microscopy, and not enabled by TEM and other technologies. Low magnification low voltage SEM, as well as VPSEM, enable stem cell biologists from all branches of Medical Sciences to bridge the gap in experimental approaches to understand cell shape, cell surface and attachment features, and is imperative before embarking on higher resolution technologies and further application. 
References

[1] E.O. Wilson, Biophilia. Harvard University Press, Cambridge, England. 1984.

[2] E.M. Hansson et al. Stem Cell Cell 5 (2009) 364.

[3] R. Passier et al. Nature 453 (2008) 322.

[4] M. Diensthuber et al. JARO 10 (2009) 173.

[5] L-M. Joubert, Microsc. Microanal. 15 (Suppl.2) 1308CD (2009).

[6] I gratefully acknowledge all collaboration on Stem Cell projects with groups at Stanford University: Aaron James, Ben Levi (Michael Longaker, PI), Oscar Abilez, Josh Baugh (Ellen Kuhl, PI), Marc Diensthuber, Kazuo Oshima (Stefan Heller, PI)

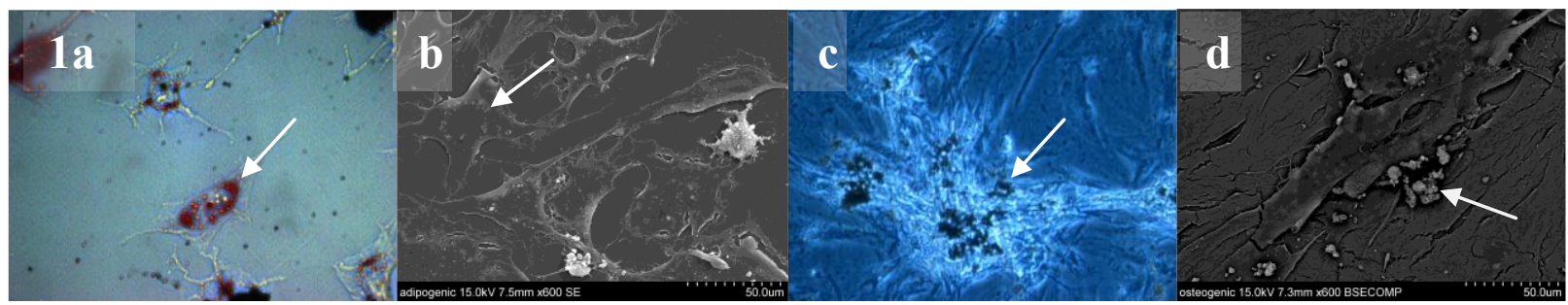

FIG.1 (a,b) Adipogenic Stem Cells (ASC, from fat cells) as seen with (a) Light Microscopy, (b)SEM (c,d) Osteogenic stem cells (with bone nodules) visualized with (c) Light Microscopy, (d) SEM BSE detection. Arrows indicate lipid droplets $(\mathrm{a}, \mathrm{b})$ and bone nodules $(\mathrm{c}, \mathrm{d})$

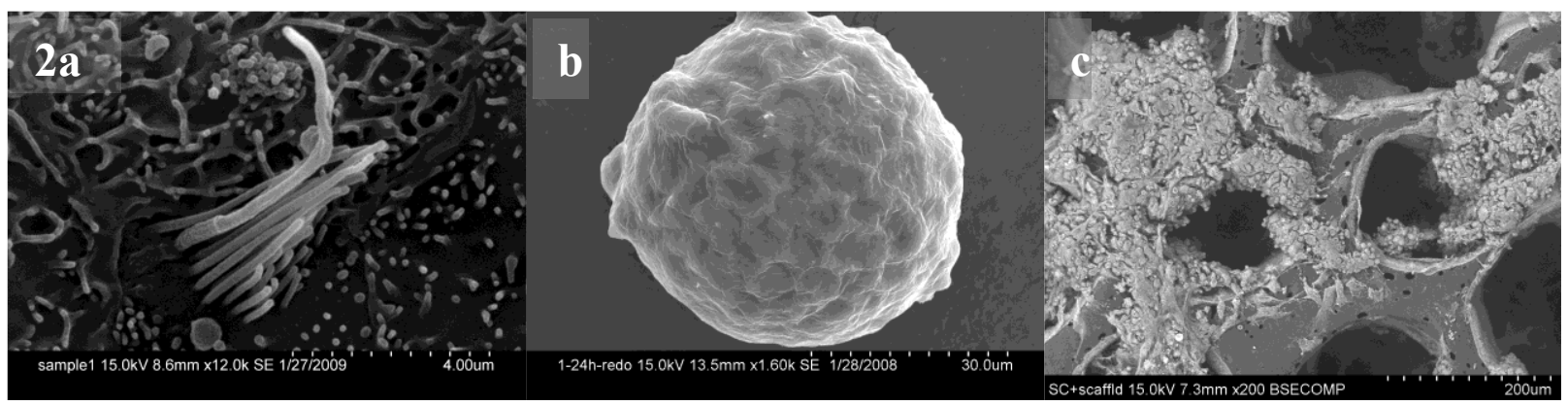

FIG.2 (a) Stereocilia from HESC, (b) Neurosphere (cochlear Stem Cell clusters) (c) ASC on PLGA scaffold visualized fully hydrated and osmicated, with VP-SEM.

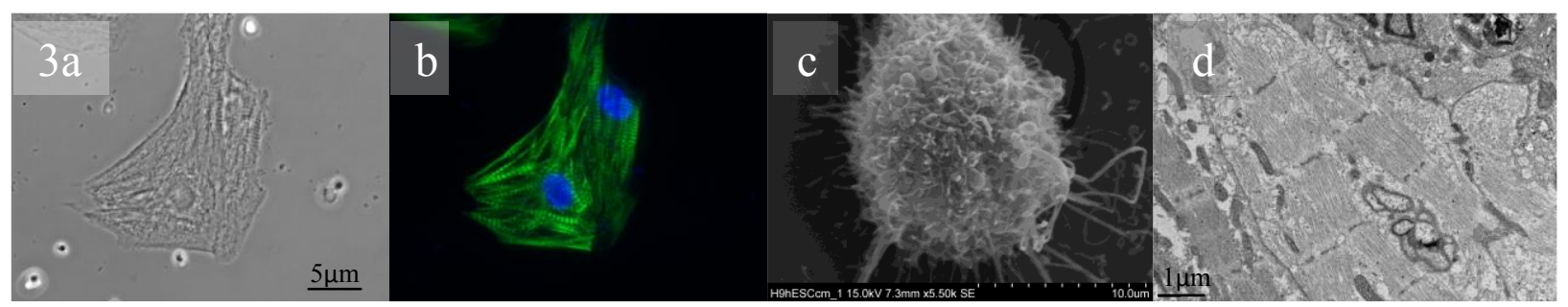

Fig.3 Cardiomyocytes as seen with (a) Light microscopy, (b) Fluorescence Microscopy, (c) SEM, (d) TEM. SEM here provides the main technology to visualize surface features. 